Apidologie, 1977, $8(3), 237-257$.

\title{
DIE BEDEUTUNG DER REBEN (VITIS spp.) ALS POLLENSPENDER FÜR DIE HONIGBIENE (APIS MELLIFICA)
}

\author{
L'importance de la vigne (Vitis spp.) pour l'abeille \\ (Apis mellifica) en tant que plante pollinifère
}

\author{
Günther VORWOHL \\ Universität Hohenheim, Landesanstalt für Bienenkunde, \\ Postfach 106-41000 D - 7000 Stuttgart 70
}

\author{
SUMMARY \\ THE IMPORTANCE OF THE GRAPE-VINE (Vitis SPP.) \\ AS POLlen-plant for the honeybee (Apis mellifera l.)
} bees :

It was possible to document in various manners that vine flowers are visited by honey-

1. by proving Vitis-pollen in the hody hair and the alimentary canal of bees which had been killed by application of pesticides in vineyards.

2. by proof of Vitis-pollen in the colonies' pollen-stores

3. by investigation of pollen traps in the grape-vine regions of South-Baden, Pfalz and Rheingau (G.F.R.)

4. by proof of Vitis-pollen in honeys

5 . by field observations.

Nectar secretion of vine flowers could not be ascertained. Pollen gathering flight generally is of such little intensity that continuous observation is necessary to become aware of it. At a few days only, the visiting flights reach high frequences (maximum $=28$ bees per vine and hour).

The investigations of pollen traps showed that, at some places and for a short time, the vines were furnishing the major part of the collected pollen.

In 1970 the bees' visits of the vines were particularly intensive. This was confirmed especially by the analysis of microscopic honey investigations. The pollen yield of one vine inflorescence of the variety "Bacchus" amounted to $1 \mathrm{mg}$. So, $16 \mathrm{~g} / \mathrm{ha}$ may be calculated. Sometimes, the vineyards are visited by bees over a distance of several kilometers. Vitis vinifera is - under the climatic and geographic conditions in the German Federal Republic a pollen plant of very little, sometimes, however, of secondary importance. 


\section{ZUSAMMENFASSUNG} werden :

Der Beflug der Rebblüten durch die Bienen konnte auf verschiedene Weise dokumentiert

1. durch Nachweis von Vitis-Pollen im Haarkleid und im Darmkanal von Bienen, die durch Pflanzenschutzmaßnahmen in Weinbergen getötet wurden, 2. durch Nachweis von Vitis-Pollen in den Pollenvorräten, 3. durch Pollenfallenversuche in den Weinbaugebieten Südbadens, der Pfalz und des Rheingaus, 4. durch Nachweis von Vitis-Pollen in Honigen, 5. durch Freilandbeobachtungen. Nektarausscheidung der Rebblüten konnte nicht festgestellt werden. Der Beflug auf Pollen ist im allgemeinen so wenig intensiv, daß es ausdauernder Beobachtung bedarf, um ihn zu bemerken. Nur an wenigen Tagen erreicht der Beflug hohe Frequenzen (maximal 28 Bienen pro Rebstock und Stunde). Anhand der Pollenfallenversuche ließ sich zeigen, daß die Reben an einigen Standorten für kurze Zeit die Hauptmenge des eingetragenen Pollens liefern. In einem Jahr (“ Flugjahr " 1970) war der Besuch der Reben besonders intensiv. Dies ergab sich besonders bei der Auswertung mikroskopischer Honiguntersuchungen. Der Pollenertrag eines Blütenstandes (Gescheins) der Rebsorte Bacchus liegt in der Größenordnung von $1 \mathrm{mg}$. Daraus läßt sich ein Hektarertrag von $16 \mathrm{~g}$ errechnen. Die Weinberge werden manchmal über eine Distanz von mehreren Kilometern angefogen. Vitis vinifera ist auch unter den klimatischen und geographischen Bedingungen der Bundesrepublik Deutschland ein Pollenspender sehr geringer, manchmal jedoch von sekundärer Bedeutung.

\section{A. - EINLEITUNG}

Bis vor wenigen Jahren war man in der Bundesrepublik Deutschland der Ansicht, daß die Weinberge während der Rebblüte von den Bienen praktisch nicht besucht werden oder wenn, dann nur wegen attraktiver Unkräuter oder Unterkulturen. Schwere Bienenschäden im Zusammenhang mit Pflanzenschutzmaßnahmen im Weinbau im Jahre 1970 (BuchNer, 1970), die besonders in Südbaden auftraten, machten es notwendig, sich mit dieser Frage intensiver zu befassen. Nach Angaben von Herrn Dr. Gnädinger (1975) beliefen sich die Schäden durch Bienenvergiftungen in Weinbergen in den fünf Jahren von 19701974 im Landesverband Badischer Imker auf 2.4 Mill. DM. Durch Nachweis von Giftrückständen im Bienentotenfall * und durch den Nachweis von VitisPollen im Haarkleid und im Verdauungstrakt der vergifteten Bienen sowie in den Pollenvorräten geschädigter Völker konnte es als erwiesen gelten, daß die Bienen unmittelbar durch das Eintragen von vergifteten Rebpollen zu Schaden gekommen waren (VorwoнL, 1970). Verluste durch Pflanzenschutzmaßnahmen an den Reben waren schon lange vor 1970 bekannt geworden (z.B. Geinitz, 1935). Man hatte sie jedoch mit Abdrift, Begiftung von Unterkulturen oder Unkräutern erklärt.

In dieser Arbeit wird der Beflug der Reben durch die Bienen auf verschiedene Weise zu dokumentieren versucht und zwar zunächst mit Laboruntersuchungen : Erstens, wie schon erwähnt, durch Nachweis von Vitis-Pollen im Haarkleid und im Darmkanal von vergifteten Bienen, zweitens durch

* durch Herrn Prof. Dr. Stute in Celle. 
Pollenfallenversuche an Völkern, die in der Nähe von Weinbergen standen, drittens durch Nachweis von eingetragenem Vitis-Pollen, insbesondere bei mutmaßlich giftgeschädigten Völkern, und schließlich viertens durch Nachweis von Vitis-Pollen in Honigen. Durch Freilandbeobachtungen sollte geklärt werden, ob, wann und möglicherweise auch warum die Bienen die Rebblüten befliegen.

\section{B. — LABORUNTERSUCHUNGEN, MATERIAL, METHODIK UND ERGEBNISSE}

\section{I. - Vitis-Pollen im Haarkleid und im Verdauungstrakt von Bienen}

In den Jahren 1970 bis 1976 gingen zahlreiche Proben toter Bienen ein, bei denen Pflanzenschutzmaßnahmen in den Weinbergen als Schadensursache vermutet wurden.

Insgesamt waren es 89 Bienenproben. Bei 61 dieser Proben wurde sowohl der Pollenbestand des Haarkleides wie auch der Polleninhalt des Darmes auf Vitis untersucht, beim Rest der Einsendungen führten wir nur eine der beiden Untersuchungen durch.

Die Gewinnung des Pollens aus dem Haarkleid erfolgt durch Waschen des Totenfalls (beliebige Menge) mit Propanol-2 (siehe auch Lukoschus, 1957). Falls die Bienen Höschen tragen, werden diese entfernt und gesondert untersucht. Die Propanol-Menge wird so bemessen, daß die toten Bienen schwimmen und der Pollen durch Schwenken aus dem Haarkleid geschwemmt werden kann. Die Propanol-Aufschwemmung wird in geeignete Zentrifugengläser gefüllt und anschließend 5 Min. bei ca. 1800 g zentrifugiert. Dann dekantiert man das Propanol vom Bodensatz, nimmt den Bodensatz mit Wasser auf und zentrifugiert erneut. Nach Abgießen des Wassers wird das Sediment auf Objektträger übertragen und nach dem Trocknen auf einer Wärmplatte mit Kaiser's Glyceringelatine eingedeckt. Die mikroskopische Untersuchung erfolgt bei 320 - und 800 -facher Vergrößerung. Die Häufigkeiten der Pollenarten werden geschätzt, vorteilhaft basierend auf der Auszählung von 100 Pollenkörnern. Häufigkeitsangaben : vereinzelt $<3 \%$, selten $3-15 \%$, häufig $16-45 \%$, sehr häufig über $15 \%$.

Da die eingesandten Bienenproben im allgemeinen während des Versandes nach Hohenheim in Verwesung übergegangen waren, wurde zur Gewinnung des Pollens aus dem Darmtrakt nich der Darm gezogen, sondern der ganze Hinterleib in 3 - 4 Stücke zerschnitten. Man wäscht die Hinterleibsabschnitte mit Wasser aus und gießt die Aufschwemmung zur Entfernung der groben Partikel durch ein engmaschiges Sieb. Die durchgelaufene Flüssigkeit wird zentrifugiert, die überstehende Lösung dekantiert und verworfen. Den Bodensatz nimmt man erneut mit Wasser auf und zentrifugiert und dekantiert nochmals. Ein Teil des Bodensatzes wird nun auf Objektträger übertragen und mit Glyceringelatine montiert. Auswertung wie oben beschrieben. Auszählungen sind schwierig, weil die Verdauung die Pollenkörner deformiert und zum Teil unbestimmbar macht (s. Allg. deutsch. Imkerztg. 1970 (9) 252).

In 69 von insgesamt 70 Präparaten von Pollen aus dem Haarkleid wurde Vitis-Pollen gefunden $(19 \times$ sehr häufig, $38 \times$ häufig, $6 \times$ selten und $6 \times$ vereinzelt).

In 72 von insgesamt 83 Untersuchungen des Darminhalts war das Ergebnis positiv für Vitis $(41 \times$ sehr häufig, $16 \times$ häufig, $11 \times$ selten und $4 \times$ verein- 
zelt). Mit einer Ausnahme brachte die gleichzeitige Untersuchung des Darms und des Haarkleides in beiden Fällen übereinstimmend positiven oder negativen Befund. Die Bienenproben mit positivem Befund stammen aus zahlreichen Ortschaften. Vielfach liegen positive Befunde für den gleichen Platz aus mehreren Jahren vor, oder für mehrere Imker, die am gleichen Ort arbeiten, oder für Parallel-Proben aus Völkern des gleichen Standes.

Die Standorte mit positiven Befunden für Vitis-Pollen liegen alle im mittel- und südbadischen Raum.

\section{II. - Vitis-Pollen in Pollenfallen}

An insgesamt 14 Bienenständen (siehe Tab. 1) wurden ein oder zwei Bienenvölker zu Beginn der Rebblüte mit Fallen versehen.

Tав. 1. - Charakterisierung der Standorte für die Pollenfallenversuche.

TABL. 1. - Caractéristiques des lieux d'expériences avec trappes à pollen.

\begin{tabular}{|c|c|c|c|}
\hline Lieu & $\begin{array}{l}\text { Entfermung zum } \\
\text { nächstgelegenen } \\
\text { Weinberg } \\
\text { Distance au vignoble } \\
\text { le plus proche }\end{array}$ & $\begin{array}{l}\text { Rebfläche innerhalb } \\
\text { eines Flugradius } \\
\text { von } 2 \mathrm{~km} \\
\text { Surface de vignes } \\
\text { a vintérieur d'un rayon } \\
\text { de vol de } 2 \mathrm{~km}\end{array}$ & $\begin{array}{l}\text { Summe der Winkel, in } \\
\text { denen die Rebanlagen, } \\
\text { vom Bienenstand aus } \\
\text { gemessen, liegen } \\
\text { Augle autour de la ruche } \\
\text { dans lequel } \\
\text { sont situées les vignes }\end{array}$ \\
\hline Freiburg $\ldots \ldots \ldots \ldots \ldots$ & $<100 \mathrm{~m}$ & $40 \mathrm{ha}$ & $148^{\circ}$ \\
\hline Badenweiler $\ldots \ldots \ldots \ldots \ldots$ & $300 \mathrm{~m}$ & $102 \mathrm{ha}$ & $98^{0}$ \\
\hline Laufen..$\ldots \ldots \ldots \ldots$ & $250 \mathrm{~m}$ & $204 \mathrm{ha}$ & $345^{\circ}$ \\
\hline Grunern $\ldots \ldots \ldots \ldots \ldots$ & $1750 \mathrm{~m}$ & 11 ha & $25^{0}$ \\
\hline Staufen $P \ldots \ldots \ldots \ldots$ & $<100 \mathrm{~m}$ & 120 ha & $322^{\circ}$ \\
\hline Staufen $\mathbf{B} \ldots \ldots \ldots \ldots$ & $250 \mathrm{~m}$ & 75 ha & $195^{\circ}$ \\
\hline Pfaffenweiler $\ldots \ldots \ldots \ldots \ldots$ & $250 \mathrm{~m}$ & 400 ha & $320^{\circ}$ \\
\hline Glottertal .......... & $<100 \mathrm{~m}$ & $62 \mathrm{ha}$ & $199^{\circ}$ \\
\hline Buchholz . . . . . . . . . & $1800 \mathrm{~m}$ & 3 hat & $6^{0}$ \\
\hline Achkarren $\ldots \ldots \ldots \ldots \ldots$ & $300 \mathrm{~m}$ & 14 I ha & $155^{\circ}$ \\
\hline Burkheim $\ldots \ldots \ldots \ldots$ & $100 \mathrm{~m}$ & $396 \mathrm{hat}$ & $290^{\circ}$ \\
\hline Durbach $\ldots \ldots \ldots \ldots \ldots$ & $<100 \mathrm{~m}$ & 109 ha & $300^{\circ}$ \\
\hline Neustadt $/ \mathbf{W}$ s......... & $<100 \mathrm{~m}$ & 742 ha & $360^{\circ}$ \\
\hline Geisenheim /Rhg. . . . . . & $100 \mathrm{~m}$ & $266 \mathrm{ha}$ & $160^{\circ}$ \\
\hline
\end{tabular}

Mit Ausnahme der Stände in Neustadt/Weinstraße und Geisenheim/ Rheingau lagen alle Versuchsstände im badischen Gebiet. Ein Teil der Versuche konnte zwei oder drei Jahre hintereinander durchgeführt werden. 
Die folgenden Parameter dienten zur Charakterisierung der Versuchsplätze hinsichtlich der Verfügbarkeit von Vitis-Pollen :

a) Die Entfernung zwischen Versuchsvolk und dem nächstgelegenen Weinberg,

b) die Größe der Rebanlagen im Flugbereich in Hektar,

c) die Summe der Winkel, in denen die Rebgelände, vom Bienenstand aus gesehen, liegen.

Zugrundegelegt wurde ein Flugradius von $2 \mathrm{~km}$. Die Auswertungen erfolgten mit Hilfe der Meßtischblätter der Versuchsgebiete (topographische Karte $1: 25000$ ).

Aufarbeitung von Höschen aus Pollenfallenversuchen.

Es wurden Pollenfallen der Fa. Thomas verwendet. Die Fallen arbeiten mit Lochstreifen nach Bötтcher. Das Sammelgut entleerte man in ein- oder mehrtägigen Abständen. Alle Versuchsstände lagen weit von Hohenheim entfernt. Die geernteten Höschen wurden von Zeit zu Zeit nach Hohenheim übersandt.

Das beste Verfahren zur quantitativen Auswertung von Pollen aus Pollenfallenversuchen ist die Sortierung der Höschen nach Pollenart, wobei insbesondere die Farbe, aber auch die Art der Packung, Oberfläehenbeschaffenheit usw. hilfreich sind. Nach dem Sortieren wiegt man die einzelnen Fraktionen aus (siehe auch Louveaux, 1958). Dies Verfahren konnte nur selten angewandt werden. Die Ernte aus den Fallen gelangte auf dem Postweg nach Hohenheim. Die Höschen waren oft zerdrückt und miteinander verklebt. Aber auch bei einwandfreier Beschaffenheit der Höschen erwies sich die Sortierung nach Farbe als schwierig. Der Pollen von Vitis ist hell-zitronengelb. Gelb in den verschiedensten Abstufungen ist aber entschieden die häufigste Pollenfarbe. Bei gleichzeitigem Vorkommen von Castanea sativa-Höschen oder solchen von Senf, Hederich oder Mädesüß wird die Sortierarbeit mühsam, entsprechend waren die (Vitis-Fraktionen ) selten ganz sortenrein.

In der Mehrzahl der Fälle wurde bei den Höschen aus Pollenfallen nur eine Teilfraktionierung vorgenommen, derart, daß man die gelben Höschen aller Schattierungen insgesamt auslas und den nicht-gelben gegenüberstellte. Waren die Pollenhöschen verklebt oder stark zerkrümelt, wurde eine Stichprobe des Materials ohne jede Fraktionierung zur Untersuchung verwendet.

Das Pollenmaterial übergießt man zur näheren mikroskopischen Untersuchung zunächst in Zentrifugengläsern mit Wasser und zerrührt dann vorsichtig. Der Zusatz eines Haushaltsspülmittels (1 Tropfen) erwies sich als vorteilhaft. Die Aufschwemmung wird abzentrifugiert und die überstehende Lösung abgegossen. Dadurch entfernt man die Zucker, mit denen die Pollen in den Höschen verklebt sind. Zur Entfernung des Pollenöls wird der Bodensatz nun mit Propanol-2 aufgenommen. Man zentrifugiert erneut und dekantiert das Propanol. Schließlich wird der Bodensatz noch einmal mit Wasser aufgenommen. Nach dem Zentrifugieren gießt man den größten Teil des Wassers ab. Mit dem Rest rührt man das Sediment auf und fertigt davon einen oder mehrere Ausstriche auf Objektträgern an. Eindecken und Mikroskopie wie unter B. I. angegeben.

Wurden die Höschen nach Pollensorten sortiert und ausgewogen, kann der Anteil der verschiedenen Pollenarten in Gewichtsprozenten angegeben werden, oder nach Auszählung in Prozent der Höschenzahl.

Bei Aufarbeitung ohne Fraktionierung kann nur der Prozentsatz der verschiedenen Pollenarten in den mikroskopischen Präparaten angegeben werden. Dabei treten die kleinen Pollenarten relativ stärker hervor, da ein Höschen oder eine Gewichtseinheit je nach Größe der Pollenart unterschiedliche Anzahlen von Pollenkörnern enthält.

Bei der überwiegenden Mehrzahl der Pollenfallenauswertungen wurde teilfraktioniert in " gelb " und “ nicht gelb ). Beide Fraktionen wurden mikroskopisch untersucht und die Häufigkeit wie unter B. I. geschätzt. Ferner wurde der Anteil der “ gelben " Höschen an den Höschen insgesamt geschätzt oder an einer Stichprobe ausgezählt. Die Häufigkeiten im 


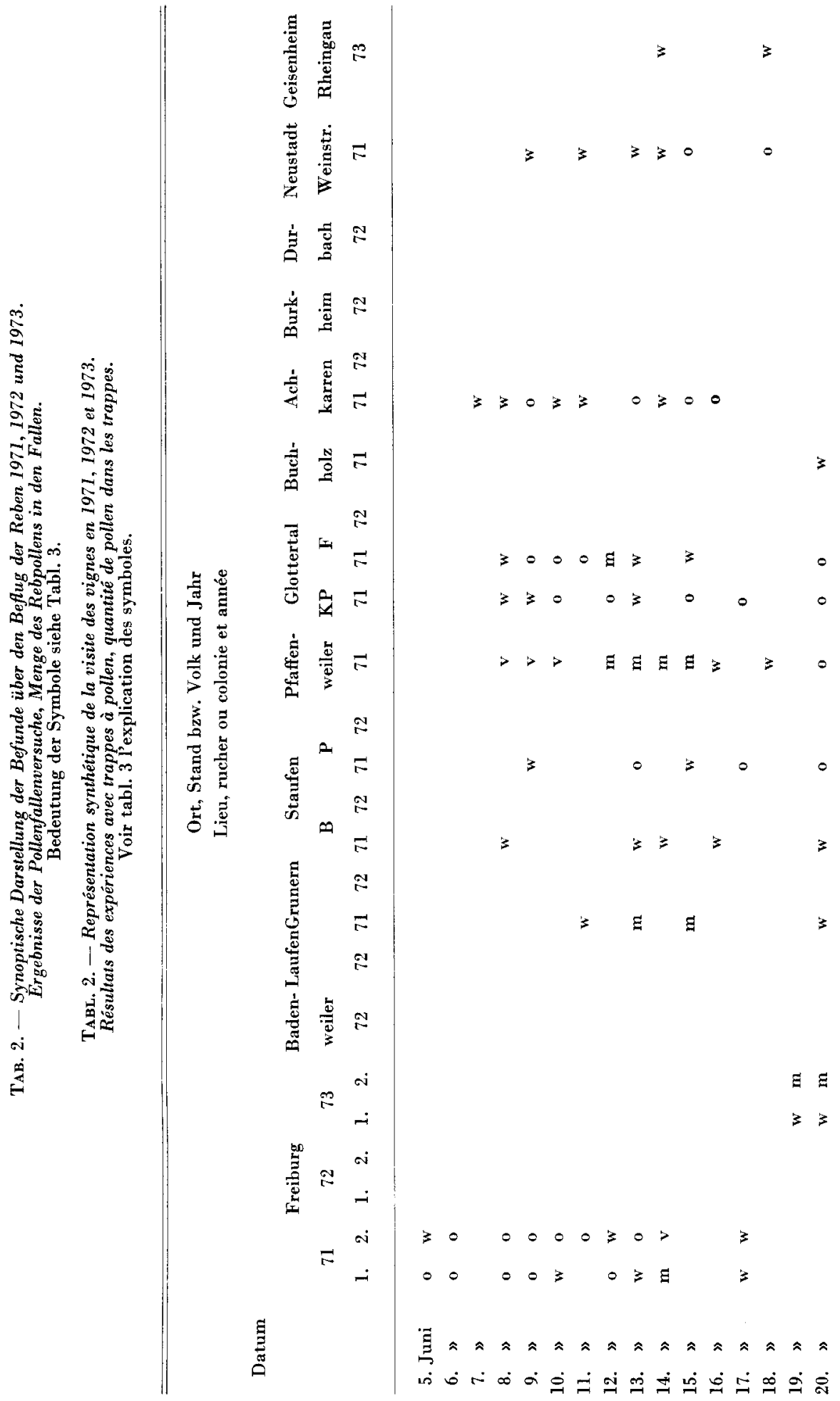


$00 \geqslant \geqslant 0$

$$
\forall 0 \#>a>>>>\text { I }
$$

- 00

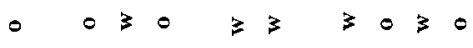

$3 \quad>$

$$
0000 \leqslant 000
$$

3

\begin{tabular}{|c|c|c|c|c|c|}
\hline 3 & 3 & 33 & 3 & 3 & 3 \\
\hline & 3 & 33 & 3 & 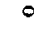 & 0 \\
\hline
\end{tabular}

$$
\begin{aligned}
& 0000 \geqslant>P>>B 0 \geqslant 000000 \\
& 000>3 E>E>>0 ; 0000
\end{aligned}
$$

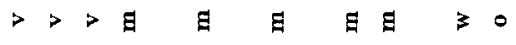

$$
\begin{aligned}
& 0000000000
\end{aligned}
$$

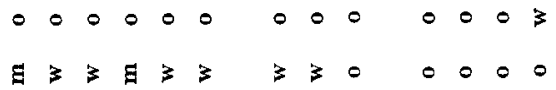

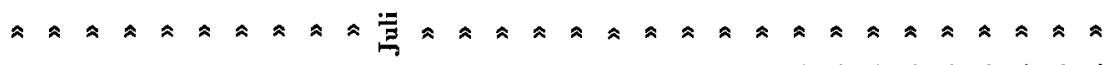

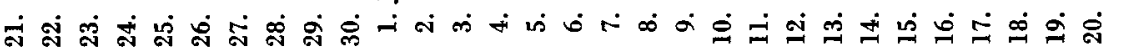


mikroskopischen Präparat der gelben Fraktion (vereinzelt, selten, häufig, sehr häufig) werden mit 1, 2, 3 und 4 bewertet (Häufigkeitsziffer), der Anteil der gelben Fraktion an der Gesamtmenge wird als Dezimale ausgedrückt (Mengenziffer). Multipliziert man beide Zahlen miteinander, ergibt sich der Mengenindex, Beispiel : In der gelben Höschenfraktion ist der Vitis-Pollen sehr häufig. Es ergibt sich also die Häufigkeitsziffer 4. Die gelbe Fraktion macht $1 / 10$ der Gesamternte aus. Die Mengenziffer ist also 0.1. Der Mengenindex errechnet sich zu 0.4. Der höchstmögliche Index ist demnach 4 (die untersuchten Höschen sind alle gelb, die mikroskopische Untersuchung ergibt mehr als $45 \%$ Rebpollen im Präparat). In den Tabellen werden die folgenden Symbole verwendet :

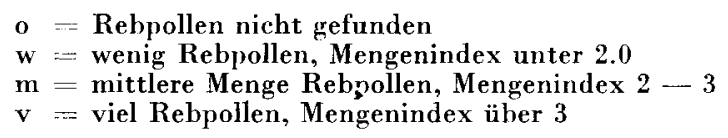

\section{Ergebnisse}

Für alle Standorte konnte nachgewiesen werden, daß die Bienen die Reben befliegen (siehe Tab. 2). Nur in einem Fall - Freiburg 1972 Volk 1 - konnte kein Rebpollen festgestellt werden. In der Mehrzahl der Beobachtungstage ist der Anteil der Reben an der Gesamternte gering. Das schließt aber nicht aus, daß Vitis an einigen Tagen einen erheblichen Anteil der Pollenernte liefert, wenn auch nicht an allen Orten und mit Unterschieden von Jahr zu Jahr und auch zwischen den Völkern eines Standes (siehe Tab. 2, insbesondere Standort Freiburg). Ähnliche Beobachtungen machten Battaglini und Ricciardeldi D'Albore (1970) bei Perugia, Italien. Ma urizio (1953) verzeichnet für Wädenswil in der Schweiz geringfügigen Vitis-Anteil bei Fallenversuchen. In 2 Beobachtungsjahren und bei Aufstellung von 2 Versuchsvölkern ergab sich nur in einem Jahr für ein Volk ein Vitis-Anteil unter $1 \%$. Sehr starken Beflug der Reben auf Pollen verzeichnet indessen Stechenкo (1958) in Rebanlagen (Sorten Saperava und Nimrang) in Kasachstan. 68-84\% der Pollenhöschen der Bienen stammten von den Rebblüten. Nach Angabe der Autoren blühten während des Versuchszeitraums wenig Konkurrenzpflanzen, eine Konstellation, die sich in den europäischen Weinbaugebieten selten ergeben dürfte. Stechenko führte zudem Duftdressuren durch, die die Bienen speziell auf die Reben hinlenken sollten. Für die Konkurrenzpflanzen wurden negative Dressuren mit vergällten Lösungen durchgeführt.

Es wurde versucht, die Pollenfallenversuche so auszuwerten, daß sie über das Konkurrenzverhältnis zwischen Rebpollen und anderen Pollenarten etwas aussagen. Dazu finden sich in Tabelle 3 die Vitis-Befunde mit der jeweils wichtigsten gleichzeitig eingetragenen Pollenart gegenübergestellt. Es zeigten sich jedoch keine übersichtlichen Beziehungen. Zum Beispiel ist die Edelkastanie, Castanea sativa, nach allgemeiner imkerlicher Erfahrung ein ausgezeichneter, ergiebiger Pollenspender. Trotzdem fand sich in vielen Fällen Vitis-Pollen zusammen mit Castanea-Pollen. Daraus den Schluß zu ziehen, die Reben seien als Pollenspender ebenbürtig zur Edelkastanie, hieße aber wohl die Ergebnisse der Fallenversuche überbewerten. Das parallele Auftreten von 
Rebpollen und Castanea-Pollen kann auch daran liegen, daß die Edelkastanie zwar hochattraktiv, aber entweder in ungenügender Menge oder zu weit vom Bienenstock entfernt angeboten wird. Ủber diese Verhältnisse vermag aber ein Pollenfallenergebnis allein keine Auskunft zu geben. Die Tabelle 3 gibt gleichzeitig eine U̇bersicht über die wichtigsten Pollenspender zur Zeit der Rebblüte.

TAB. 3. - Häufigkeit des Vitis-Pollens und wichtigste parallele Pollentracht bei den Pollenfallenversuchen

TaBL. 3. - Fréquence du pollen de Vitis et récoltes parallèles de pollen les plus importantes lors des expériences avec les trappes à pollen

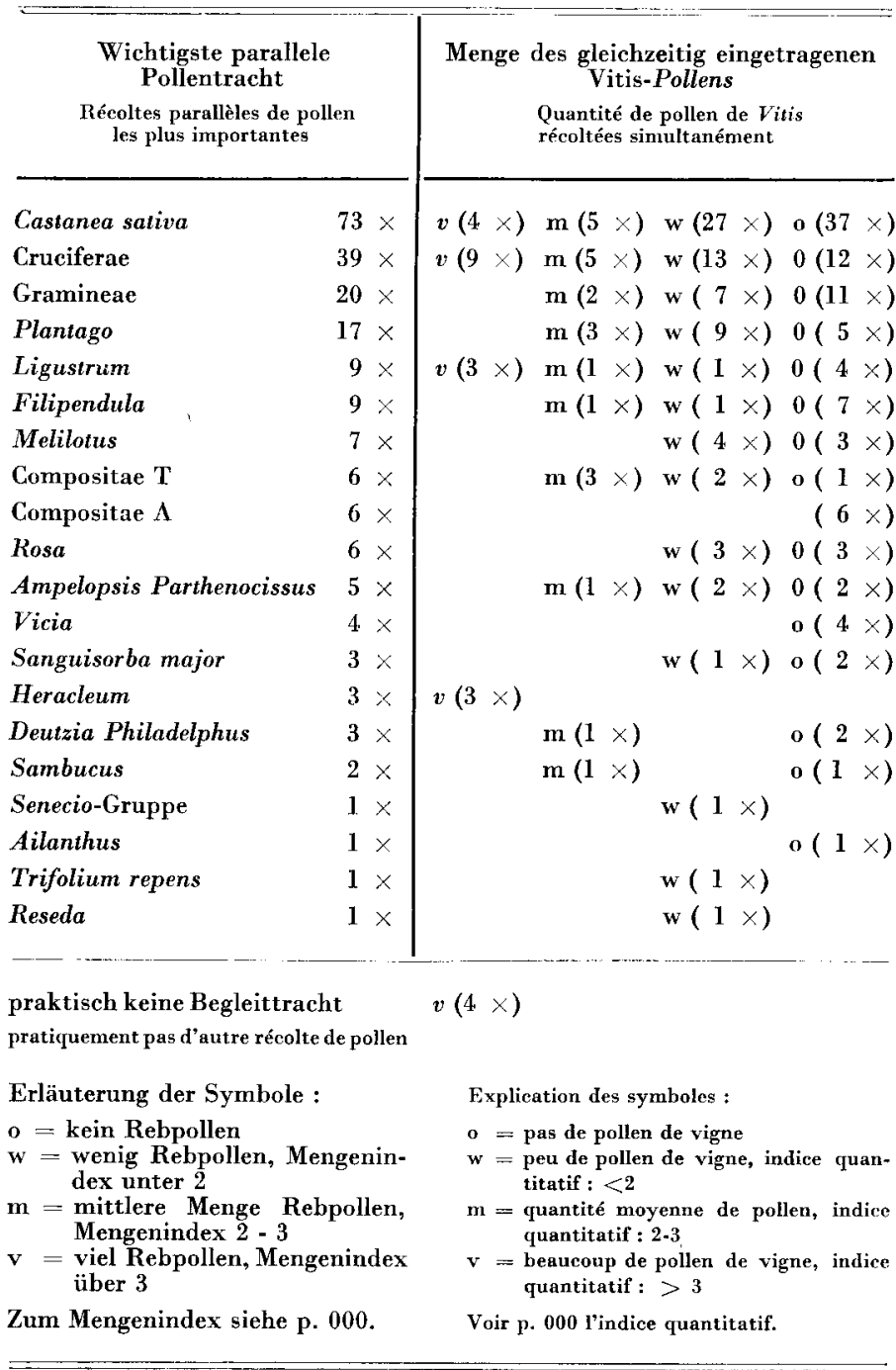


Es ist zu erwarten, daß an Standorten, an denen die Versuchsvölker fast allseits von Rebgelände umgeben sind (wie z.B. in Pfaffenweiler, Laufen, Staufen P, Burkheim und Neustadt) regelmäßiger und mehr Rebpollen eingetragen wird als an Standorten mit weniger einseitiger Flächennutzung. Die Ergebnisse unserer Beobachtungen bestätigen diese Annahme nur bedingt. So brachten die Fallenversuche in Buchholz 1971 und in Neustadt/Ws. 1971 etwa das gleiche Ergebnis, obwohl Buchholz bei der Charakterisierung der Standorte den schlechtesten Stellenwert hat (größere Rebgelände nur in einer Richtung außerhalb der $2 \mathrm{~km}$-Zone), während Neustadt an der Spitze steht (Rebgelände allseits um den Versuchsstand, $59 \%$ des Flugareals sind mit Vitis bestockt).

\section{III. - Vitis-Pollen im Bienenbrot}

In den Jahren 1970-1976 wurden insgesamt 39 Waben oder Wabenstücke mit eingelagertem Pollen (Bienenbrot) untersucht. Die Proben entstammen geschädigten Völkern, bei denen Pflanzenschutzmaßnahmen als Ursache der Verluste vermutet wurden.

Aus dem in die Waben eingelagerten Pollen wurden möglichst viele Stichproben von gelbem Bienenbrot entnommen. Die vereinigten Proben wurden wie Pollenhöschen (siehe B II) verarbeitet. Die Mengenangabe beschränkt sich auf sehr viel, viel, wenig. Genauere Quantifizierungen würden großen Arbeitsaufwand erfordern, ergäben aber nur eine Scheinexaktheit, da ein Wabenstück i $m$ allgemeinen nicht als repräsentativ für den Pollenvorrat eines ganzen Volkes angesehen werden kann.

In 34 von 39 Fällen war das Ergebnis positiv. Nur in 5 Proben konnte kein Rebpollen nachgewiesen werden. Mehrfach enthielten die Waben vorwiegend Rebpollen. Bei zwei Proben handelt es sich um « Pollenbretter ) mit Vitis-Bienenbrot, also Waben, bei denen beidseitig fast alle Zellen mit Rebpollen gefüllt waren. Dieser war also in erheblichen Mengen eingetragen worden. Mit Ausnahme von zwei Einsendungen aus Weinsberg, Reg. Bez. Nordwürttemberg, stammten alle Wabenproben aus dem badischen Weinbaugebiet.

\section{IV. - Vitis-Pollen im Honig}

Zur Methodik der mikroskopischen Honiguntersuchung siehe Louveaux, Maurizio, VoRWOHL (1970).

Ausgewertet wurden 1200 Pollenanalysen, die in den Jahren 1962-1976 routinemäßig an Honigen aus Baden-Württemberg durchgeführt wurden. Die Honigmuster stammen aus allen Teilen des Landes. Die Anzahl der Proben 
aus dem Bereich des Landesverbandes Badischer Imker liegt allerdings höher als die aus Württemberg. Im Berichtszeitraum wurden in Baden mehrere Honigprämiierungen durchgeführt, wobei die Voruntersuchung der Honige an der Landesanstalt in Hohenheim erfolgte. Ferner hatte die Landesanstalt bis 1967 eine Außenstelle in Staufen im Breisgau, die besonders von Imkern des südbadischen Raumes für Honiguntersuchungen in Anspruch genommen wurde.

Zwischen Honigen aus den Weinbaugebieten des Landes und Honigen aus den Regionen, in denen Rebstöcke nur gelegentlich als Spaliere in Gärten oder an Häusern zu finden sind, wurde nicht unterschieden.

Die Honigmuster wurden ausschließlich nach Eingangsdatum gruppiert, da sie in der Mehrzahl undatiert eingingen. Die Einteilung erfolgte nach Jahrgängen, die jeweils vom 1. Juni des notierten bis zum 31. Mai des folgenden Jahres reichen. In den einzelnen Jahrgängen fielen die folgenden Probenzahlen an :

$\begin{array}{rr}1962-33 & 1969-23 \\ 1963-49 & 1970-264 \\ 1964-158 & 1971-73 \\ 1965-51 & 1972-188 \\ 1966-45 & 1973-28 \\ 1967-81 & 1974-119 \\ 1968-80 & 1975-57\end{array}$

Die Untersuchung der mikroskopischen Präparate erfolgte im allgemeinen " orientierend ", d.h. das unter dem Deckgläschen eingeschlossene Sediment wurde nicht vollständig Bahn für Bahn durchgesehen, sondern nur so lange, bis der Formenbestand weitgehend erfaßt und die von den Einsendern gestellten Fragen beantwortet werden konnten. Sehr vereinzelt vorkommende Vitis-Pollen können bei diesem Verfahren also übersehen werden. Mit wenigen Ausnahmen erfolgten die Untersuchungen nicht mit dem speziellen Ziel, Vitis-Pollen zu finden.

Die Ergebnisse sind in Abb. 1 zusammengefaßt. Besonders fällt das gehäufte Auftreten von Vitis-Pollen im Jahrgang 1970/71 auf. Offensichtlich wurden die Reben in diesem Jahr ungewöhnlich massiv beflogen. Nicht nur die Anzahl der Honigmuster mit positivem Vitis-Befund war hoch. Der VitisPollen erreichte auch in einzelnen Proben ungewöhnlich hohe Prozentsätze $(1 \times$ sehr häufig, d.h. über $45 \%$, und $6 \times$ häufig, d.h. $16-45 \%)$. Weder in den acht Jahren vor 1970 noch in den fünf Jahren danach wiederholte sich eine solche Konstellation. Man muß also mit besonderen, in größeren Abständen auftretenden " Flugjahren " rechnen, in denen die Reben besonders intensiv von den Bienen besucht werden. Über die Ursache dieser “ Flugjahre » können nur Vermutungen geäußert werden. An den Fakten als solchen besteht 
aber kein Zweifel. Für den Pflanzenschutz sind diese Ausnahmejahre von besonderer Bedeutung. Das “ Flugjahr » 1970 war gleichzeitig das Jahr der stärksten Bienenschäden durch Pflanzenschutzmaßnahmen in den Weinbergen. Der erfreuliche Rückgang der Schadensfälle in den Jahren 1972 bis 1975 wurde möglicherweise begünstigt durch die gegenüber 1970 geringere Tendenz zum Beflug der Reben in diesen Jahren. Die Vorbeugungsmaßnahmen sollten so beschaffen sein, daß sie auch in Ausnahmejahren wie 1970 ausreichen. 1976 scheint wiederum ein " Flugjahr " gewesen zu sein und brachte auch erhebliche Schäden.

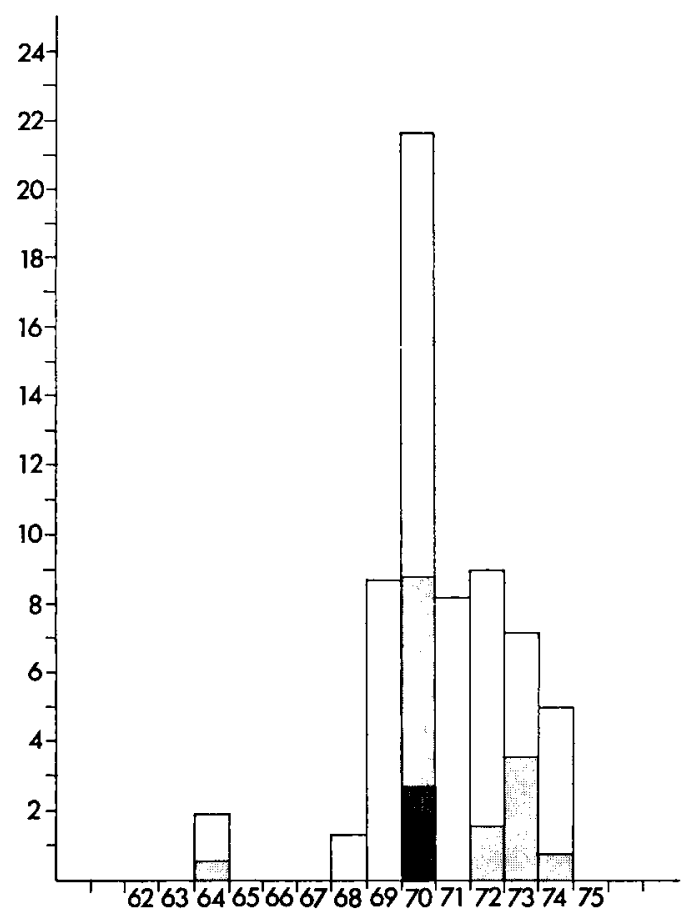

Авв. 1. - Vorkommen von Vitis-Pollen in Honigen aus Baden-Würtemberg in den Jahren 1962-1975.

Ordinate: Prozentsatz der Honige mit Vitis-Pollen.

Abszisse : Eingangsdatum der Honigproben nach Jahrgängen (jeweils vom 1. Juni des notierten bis 31. Mai des folgenden Jahres).

Häufigkeit des Vitis-Pollens in den positiven Fällen : schwarze Säule - über $45 \%$, Säule in dunklem Grauton - 16-45\%, Säule in hellem Grauton - 3-15\%, weiße Süule - unter $3 \%$ \%.

Fig. 1. - Présence de pollen de Vitis dans des miels du Bade-Würtemberg récoltés entre 1962 et 1975.

En ordonnées : Pourcentage des miels contenant du pollen de Vitis

En abscisses : Date d'entrée des échantillons de miel par année (période allant du 1 er juin au 31 mai de l'année suivante).

Fréquence du pollen de Vitis dans les cas positifs:

colonne noire : > $>5 \%$

colonne gris foncé : 16-45\%

colonne gris clair : $3-15 \%$

colonne blanche : $<3 \%$ 
Auffällig ist ferner, daß in den Jahrgängen 1962-1967 die Zahl der Proben mit positivem Vitis-Befund gering ist. Von insgesamt 417 Honigen enthielten nur 3 (also $0.7 \%$ ) selten oder vereinzelt Vitis-Pollen. Für die Jahrgänge 19681975 sind durchschnittlich $7.6 \%$ positive Befunde zu verzeichnen bei einer Probenzahl von insgesamt 832 Honigen. Ein Teil der positiven Befunde für die Jahrgänge 1971-1975 dürfte noch auf Honige der Ernte 1970 zurückgehen, die erst in späteren Jahren zur Untersuchung kamen.

Insgesamt darf man aber von einer zunehmenden Tendenz hinsichtlich der positiven Vitis-Befunde in Honigen aus Baden-Württemberg seit Ende der Sechzigerjahre sprechen. Bisher fehlen lediglich für den Jahrgang 1975/76 positive Vitis-Befunde. 1976/77 wird man mit einiger Sicherheit wieder VitisPollen in den Honigen finden.

Eine der Ursachen könnte in der Zunahme der Rebflächen liegen. 1959 waren im Landesteil Baden 5938 ha mit Reben bestockt und in Württemberg $6756 \mathrm{ha}$.

1970 lauten die entsprechenden Zahlen 9525 ha und 6258 ha.

1975 umfaßten die Ertragsweinberge in Baden 13039 ha und im Landesteil Württemberg 7603 ha.

Die Zunahme ist allerdings nur relativ. Vor dem 2. Weltkrieg beliefen sich die Rebflächen in Baden auf 12077 ha und in Württemberg auf 10207 ha. Von der Fläche her betrachtet wären also auch schon vor dem Krieg positive Befunde für Vitis-Pollen zu erwarten gewesen.

Die Rebanlagen haben sich jedoch in der Zwischenzeit verändert und sind ärmer an Pollen- und Nektar-Spendern geworden, bedingt durch die Flurbereinigung, der viele Trockenmauern, kleine Brachflächen und Hecken zum Opfer fielen.

Bis 1952 waren nur 100 ha als flurbereinigt registriert. Bis $1962 \mathrm{nahm}$ die Zahl auf 4300 ha zu, 1970 auf 8567 ha und 1975 auf 11865 ha. Insgesamt wurden bisher also ca. $58 \%$ der Rebfläche erfaßt. Ein wichtiger Faktor dürfte die raschere Mahd der Wiesen sein. Die Wiesenblüte und die Rebblüte fallen zeitlich meist zusammen. Durch die raschere Mahd verschwinden plötzlich reiche Pollenquellen, was die Attraktivität der Reben relativ steigert.

Aufgrund der Erfahrungen Zanders und späterer Untersucher (Maurizio 1956) wertete man Vitis-Pollen in Honigen, die als einheimisch deklariert waren, als Verdachtsmoment für ausländische Herkunft, da authentische deutsche Honige, soweit sie damals untersucht waren, fast nie Rebpollen enthielten, während sie in Honigen südlicherer Länder (Tessin, Italien, Ungarn) öfter zu verzeichnen waren. Nach den Ergebnissen unserer Untersuchungen muß diese Interpretation fallen gelassen werden. 


\section{C. - FREILANDBEOBACHTUNGEN}

\section{Ortliche Voraussetzungen in Hohenheim}

Die Rebanlage des Instituts für Obst-, Garten- und Weinbau liegt in unmitéelbarer Nähe der Landesanstalt für Bienenkunde und ihrer Bienenstände. Die Anlage umfaßt 6700 Stock und ist etwa 2.25 ha groß. Die Weinberge des Neckartales und seiner Nebentäler und die Rebhänge des Stuttgarter Talkessels liegen weit entfernt (mindestens $5 \mathrm{~km}$ Luftlinie). Geht man von einem Flugradius von $2 \mathrm{~km}$ aus, so umfaßt der Flugbereich $(=1257 \mathrm{ha}) 5 \%$ Wald, $19 \%$ Wiesen und Weiden, $43 \%$ Ackerland und $33 \%$ locker bebautes Gelände mit vielen Gärten und Parks. Es handelt sich also um einen relativ kleinen Weinberg in isolierter Lage in einem Gebiet mit sehr mannigfaltiger Flächennutzung.

In geringer Entfernung vom Hohenheimer Weinberg liegen außer den Bienenständen der Landesanstalt noch 3 weitere Stände mit bis zu 12 Völkern, von denen einer 1975 aufgelöst wurde. Am Rand des $2 \mathrm{~km}$ - Bereiches im Ortsgebiet Kemnat findet sich wieder eine größere Zahl von Völkern (1972 23 Völker und Ableger, 197317 Völker).

Während der Beobachtungsperioden befanden sich auf dem Gelände der Landesanstalt die folgenden Völker :

197228 Völker in durchschnittlich 2 Magazinen I Klotzbeute

197335 Völker in durchschnittlich 1.5 Magazinen + 2 Kleinvölker

1974 66 Völker in durchschnittlich 1.2 Magazinen +12 Kleinvölkchen

1975 maximal 52 Völker in durchschnittlich 2.1 Magazinen, dazu maximal 149 Kleinvölkchen. Völkerbestand vom Beginn der Beobachtungszeit ansteigend. Bestand an Kleinvölkchen wechselnd

1976 maximal 54. Völker in durchschnittlich 1.5 Einheiten pro Volk und maximal 148 Kleinvölkchen.

Die Trachtverhältnisse sind infolge der weitgefächerten Flächennutzung schwer abzuschätzen. Großflächig angebaute Pollenspender fehlen in der Nähe der Bienenstände. Nur im Beobachtungsjahr 1975 blühte in etwas mehr als zwei Kilometer Entfernung ein Sommerrapsschlag von etwa 2 ha Größe. Die ackerbaulich genutzten Flächen sind sehr wenig verunkrautet und trachtmäßig weitgehend steril. In größerer Entfernung (Plieninger Flur) finden sich einige kleine Äcker mit Futtergemengen, die Rotklee oder Perserklee (Trifolium resupinatum) enthalten. Ein wichtiger Faktor dürften die Wiesen und Weiden sein. Ein Teil derselben, besonders im Aussenbereich der $2 \mathrm{~km}$-Zone, sind noch artenreich und werden spät oder manchmal überhaupt nicht gemäht. 1972, 1973 und 1974 fand die Wiesenmahd während der Beobachtungszeit statt, woraus sich eine Verschlechterung der Pollen- und Nektar-Tracht ergab. 1975 war die Mahd zu Beginn der Beobachtung infolge anhaltend trocken-warmen Wetters fast abgeschlossen, auch auf den Weiden blühte praktisch nichts mehr. Die anschließend feuchtere Witterung begünstigte die Wiesenblüte, sodaß die Nektar- und Pollen-Versorgung durch die Wiesen und Weiden sich 1975 im Laufe der Beobachtungszeit verbesserte.

1976 brachte während der Beobachtungszeit sehr warmes und extrem trockenes Wetter. Die Wiesenmahd war zu Beginn des Beobachtungszeitraums beendet. Pollenspender waren die Gartenanlagen, die wenigen Lindenbäume und kleine Flächen mit Futtergemenge, die infolge des fehlenden Niederschlags geringe $W$ üchsigkeit zeigten. Die Wildflora und die Wiesen waren weitgehend vertrocknet.

Die zahlreichen Gärten liefern Pollen besonders von Rosen mit weniger gefüllten Blüten und Ziersträuchern wie Philadelphus und Deutzia, Kolkwitzia sowie späten Cotoneaster-Arten.

Methodik der Beobachtung der Flugintensität.

Ein Weinstock mit einer möglichst großen Zahl blühender Gescheine wurde ausgewählt und im allgemeinen eine halbe oder eine Stunde genau auf Bienenbeflug beobachtet. Die anfliegenden Sammlerinnen wurden vom Anflug bis zum Abflug im Auge behalten. Wechselte eine Sammlerin im Überschneidungsbereich von zwei Rebstöcken hin und her, wurde nur ein Anflug gezählt. Hingegen wurde jeder Anflug von weiter her gewertet, auch wenn die beobachtete Biene möglicherweise schon einmal Blüten des observierten Stockes beflogen hatte. 
Der Beflug der Nachbarstöcke und der parallelen Reihen wurde soweit als möglich mit beobachtet. Der Protokollant saß in etwa $1 \mathrm{~m}$ Entfernung am Boden, um den Anflug der Bienen nicht zu stören.

\section{Beobachtungsergebnisse.}

Die Tabelle 4 liefert eine Übersicht über die Zahl und die Art der durchgeführten Freilandbeobachtungen (siehe auch VorwoHL 1977). Die Sitzungen mit positivem Ergebnis überwiegen eindeutig, insbesondere wenn man die Beobachtungen am Vormittag ins Auge faßt. Die Besuchsfrequenz bleibt im allgemeinen niedrig. Im normalen imkerlichen Sprachgebrauch spricht man nicht von Beflug, wenn z.B. während einer mehrstündigen Beobachtungszeit nur eine Biene den anvisierten Rebstock besucht. Wenn der Beflug auch dem weniger geduldigen Betrachter auffallen soll, muß die Intensität bei etwa 6 Bienen pro Rebstock und Stunde (oder höher) liegen. 6 Bienenbesuche in der Stunde besagt durchschnittlich eine Visite in 10 Minuten. Da ein Beobachter mehrere Stöcke gleichzeitig im Auge behalten kann und der Besuch eines Stockes im allgemeinen einige Minuten dauert, wird sich bei dieser Frequenz fast immer wenigstens eine Biene im observierten Areal an den Blüten zu schaffen machen.

ТАв. 4. -- $\dot{U}$ bersicht über die Zahl und Art der Beobachtungen über den Bienenbeflug in der Rebanlage Hohenheim

TABL. 4. - Résumé du nombre et du type d'observations faites sur la visite des vignes par les abeilles à Hohenheim

\begin{tabular}{|c|c|c|c|c|c|c|}
\hline $\begin{array}{l}\text { Versu- } \\
\text { chsjahr }\end{array}$ & $\begin{array}{l}\text { Anzahl der } \\
\text { Beobachtun } \\
\text { gssitzungen, } \\
\text { insgesamt }\end{array}$ & $\begin{array}{c}\text { Anzahl der } \\
\text { Langzeitbeo- } \\
\text { bachtungen }\end{array}$ & $\begin{array}{c}\text { Anzahl der } \\
\text { Beobach- } \\
\text { tungen am } \\
\text { Nachmittag }{ }^{1}\end{array}$ & $\begin{array}{c}\text { Anzahl der } \\
\text { positiven } \\
\text { Beobachtun } \\
\text { gen insgesamt }\end{array}$ & $\begin{array}{c}\text { davon deut } \\
\text { lich positiv } \\
\left(>6 \mathrm{~B} / \mathrm{W}_{\mathrm{s}} / \mathrm{h}\right)^{2}\end{array}$ & $\begin{array}{c}\text { Anzahl der } \\
\text { positiven } \\
\text { Nachmittags- } \\
\text { beobachtungen }\end{array}$ \\
\hline Année & $\begin{array}{l}\text { Nombre total } \\
\text { de séances } \\
\text { d'observation }\end{array}$ & $\begin{array}{c}\text { Nombre } \\
\text { d'observations } \\
\text { de longue durée }\end{array}$ & $\begin{array}{c}\text { Nombre } \\
\text { d'observations } \\
\text { faitesl'après-midi1 }\end{array}$ & $\begin{array}{l}\text { Nombre total } \\
\text { d'observations } \\
\text { positives }\end{array}$ & $\begin{array}{c}\text { dont } x \\
\text { observations } \\
\text { nettement } \\
\text { positives } \\
(<6 \mathrm{~B} / \mathrm{Ws} / \mathrm{h})^{2}\end{array}$ & $\begin{array}{c}\text { Nombre } \\
\text { d'observations } \\
\text { positives } \\
\text { l'après-midi }\end{array}$ \\
\hline 1972 & 7 & - & 2 & 5 & 1 & 1 \\
\hline 1973 & 31 & 11 & $8^{3}$ & 17 & 2 & $1^{4}$ \\
\hline 1974 & 7 & 一 & I & 3 & 0 & 0 \\
\hline 1975 & 25 & 1 & 4 & 22 & 14 & 2 \\
\hline 1976 & 10 & - & - & 7 & 2 & - \\
\hline insg. 5 & 80 & 12 & 15 & 54 & 19 & 4 \\
\hline \multicolumn{7}{|c|}{$\begin{array}{l}\text { 1. nach } 12^{23} \mathrm{MEZ} \\
\text { 2. }>6 \mathrm{~B} / \mathrm{Ws} / \mathrm{h}=\text { über } 6 \text { Bienen pro Weinstock } \\
\text { und Stunde. } \\
\text { 3. davon } 3 \text { Langzeitbeobachtungen. } \\
\text { 4. deutlich positiv. }\end{array}$} \\
\hline
\end{tabular}


Im Gegensatz zu den mehrheitlich positiven Beobachtungen am Vormittag ist die Zahl der Bienenbesuche an den Rebblüten am Nachmittag gering. Im speziellen Falle des deutlich positiven Befundes 1973 handelt es sich um einen " verschobenen Vormittagsbeflug ", bedingt durch Regen am frühen Morgen und durch starke Bewölkung und niedrige Temperaturen bis zum Mittag. Für den geringen Beflug am Nachmittag können jedoch Witterungsbedingungen nicht verantwortlich gemacht werden. Während der Beobachtungssitzungen herrschte oft schönstes Flugwetter mit lebhaftem Bienenflug bei den Völkern der Landesanstalt und intensivem Bienenbesuch auf blühenden Unkräutern oder Unterkulturen im Weinberg.

Unsere Beobachtungen, nach denen die Reben zum « Morgentyp » unter den Pollenspendern gehören, stehen im Widerspruch zu den Angaben Stechenkos (1958), nach denen Weinpollen den ganzen Tag über eingetragen wurden. Die Veröffentlichung Sтеснеnкos bezieht sich auf Versuche, die in Kasachstan durchgeführt wurden und zwar unter Bedingungen, die stark von den hiesigen abweichen (andere Rebsorten, wenig konkurrierende Trachtpflanzen, Dressur der Bienen auf die Rebblüten und negative Dressur mit vergällten Lösungen auf die Konkurrenztrachten). Hingegen stimmen unsere Ergebnisse weitgehend überein mit den Beobachtungen von Sharples et al. (1965), die für die “ Cardinal Grape ) unter californischen Bedingungen die folgenden durchschnittlichen Beflugszahlen pro Rebstock angeben. Die Zahlen gelten jeweils nur für die angegebene Uhrzeit. Es wurde an 6 Tagen eine Weinstockreihe abgelaufen und die Zahl der Bienen notiert. Die Methodik unterscheidet sich also von der unsrigen.

\begin{tabular}{r|c||c|c}
\hline Zeit & $\begin{array}{c}\text { Bienen } \\
\text { pro Rebstock }\end{array}$ & Zeit & $\begin{array}{c}\text { Bienen } \\
\text { pro }\end{array}$ \\
\cline { 2 - 4 } $7^{30}$ & 0.00 & 0.16 \\
$8^{30}$ & 0.06 & $12^{30}$ & 0.06 \\
$9^{30}$ & 0,12 & $13^{00}$ & 0.04 \\
$10^{30}$ & 0.14 & $14^{00}$ & 0.05 \\
$11^{30}$ & 0.21 & $15^{00}$ & 0.00 \\
$17^{00}$ & 0.00 \\
\hline
\end{tabular}

Unter den Hohenheimer Bedingungen zeigt sich die Tendenz zum morgendlichen Beflug am deutlichsten.

Bei den Beobachtungen im Hohenheimer Weinberg wurde nur Pollensammeln festgestellt. Keine der anfliegenden Bienen zeigte die Verhaltensweisen des Nektarsammelns.

Die Hohenheimer Anlage umfaßt eine große Zahl von Sorten der Art Vitis vinifera sowie ein Sortiment anderer Vitis-Arten und Hybriden. Dadurch 
erstreckt sich die Blütezeit über fast einen Monat. Die Bienen besuchten den Weinberg innerhalb der gesamten Blütezeit.

Die Wildarten von Vitis (V. riparia, V. aestivalis, V. rupestris) und ihre Kreuzungen, die als Unterlagenreben dienen, und die Hybriden (Landot, Bourdin) werden besser beflogen als die verschiedenen Kultur-Varietäten von Vitis vinifera. Das hängt möglicherweise damit zusammen, daß die Varietäten von Vitis vinifera seit langer Zeit nur vegetativ vermehrt werden, während die Wildarten auf die geschlechtliche Vermehrung angewiesen sind. Wahrscheinlich erzeugen sie mehr oder attraktiveren Pollen als die verschiedenen Sorten von Vitis vinifera. Die Sorte “ Aris » macht jedoch eine Ausnahme von der genannten Regel. Diese Züchtung ist eine Hybridrebe, allerdings stark mit Vitis vinifera rückgekreuzt. Da die Wildarten und Kreuzungen zwischen diesen nur als Unterlagen verwendet werden, kommen sie nur gelegentlich in Rebschulen zur Blüte. Die Hybridreben sind in der Bundesrepublik Deutschland nicht zum Anbau zugelassen.

Von allen Beobachtungsjahren zeigte 1975 den intensivsten Beflug. Das hängt wahrscheinlich mit der relativ großen Völkerzahl im Gelände der Landesanstalt zusammen und mit der Fluktuation im Bestand an Bienenvölkern. Im Jahr 1976 konnte der letztgenannte Faktor genauer beobachtet werden. Zu Beginn des Beobachtungszeitraums war der Völkerbestand stationär, 47 Völker in insgesamt 78 Magazineinheiten und 31 Klein- und Kleinstvölkchen. Während der ersten 6 Beobachtungstage war der Beflug minimal, vor Beginn der Beobachtungen am 7. Beobachtungstag wurden 9 Kirchhainer Kästchen und 1 Drohnenvolk angeliefert. Am Abend des gleichen Tages nochmals 117 Kirchhainer Kästchen und 6 Drohnenvölker. Die Zahl der Besucher an den Rebblüten stieg auf 6 und am nächsten Morgen auf 7 Bienen pro Weinstock und Stunde an, fiel aber bereits am 9. Beobachtungstag wieder auf 2 Bienen pro Stock und Stunde zurück.

1976 brachte ein unerwartet schlechtes Ergebnis. Obwohl die Hitze und Trockenheit das Pollenangebot im Gelände stark verminderte und trotz Auswahl einer Rebsorte, die sich 1975 als sehr attraktiv erwiesen hatte (Landot), war der Beflug mit Ausnahme von 2 Tagen gering. In den vorausgegangenen Jahren war allerdings schon der Eindruck entstanden, daß Dauer-Schönwetter den Beflug eher vermindert, wahrscheinlich weil es den Bienen Gelegenheit bietet, sich auf ergiebigere Quellen einzufliegen, während Schlechtwetter und der damit verbundene Pollenmangel dazu führt, daß bei Wetterbesserung zunächst alle Pollenspender genutzt werden.

Trotz relativ hoher Bienendichte war der Beflug 1974 schlecht. Die anhaltend kühle Witterung führte dazu, daß an einem Geschein nur wenige Blüten an einem Tage erblühten, während die Masse entweder noch geschlossen oder schon abgeblüht war. Dadurch vermindert sich die Attraktivität, weil 
die wenigen pollenliefernden Blüten das Absammeln kaum lohnen. An mehreren Tagen wurden keine Beobachtungen vorgenommen, weil kein Rebstock mit einer größeren Zahl frisch geöffneter Einzelblüten zu finden war.

Eine Vorhersage des Beflugs scheint nach den Erfahrungen der Freilandbeobachtungen nicht möglich zu sein. Die Zahl der beeinflussenden Faktoren ist zu groß und zum Teil schwer zu quantifizieren : Pollenbedarf der Völker, Pollenangebot im Areal, Bienendichte, Sammelgewohnheiten der Bienen (bzw. deren Störung durch Schlechtwetter oder Wanderung) und Witterungseinflüsse (Verhinderung der Nutzung des Angebots durch Kälte oder Regen).

\section{D. - BEFLUG DURCH ANDERE INSEKTENARTEN}

Neben den Bienen waren in Hohenheim Bombus-Arten die häufigsten Besucher der Rebblüten. Oft sind die Besuche dem nervösen und hastigen Sammelverhalten dieser Insekten entsprechend nur kurz. Es kommt aber auch zu systematischem Absammeln der Stockreihen mit der Bildung von Höschen aus Vitis-Pollen. Gelegentlich waren auch bauchsammelnde solitäre Bienen an den Rebblüten zu beobachten. Häufig lassen sich Schwebfliegen (Sirphidae) auf den Gescheinen nieder. Sie sind keine Pollensammler, tupfen aber die Blüten mit ihrem Rüssel ab und nehmen dabei wahrscheinlich Blütenstaub mit.

\section{E. - POLlenertrag Der REBsorte Bacchus}

Im Sommer 1976 wurden insgesamt 214 Gescheine der Rebsorte Bacchus getütet. Nach dem Abblühen wurde der Pollen ausgeklopft, von den Antherenresten und Calyptren durch feine Siebe abgetrennt und anschließend gewogen. Im Durchschnitt belief sich der Pollenertrag pro Geschein auf $1.03 \mathrm{mg}$. Nimmt man 20 Gescheine für einen volltragenden Stock an und eine mittlere Stockzahl von 8000 pro Hektar, so ergibt dies einen größenordnungsmäßigen Ertrag von $16 \mathrm{~g}$ pro Hektar. Dies ist ein recht geringer Wert, der nur dadurch teilweise ausgeglichen wird, daß die Reben in ausgedehnten Reinkulturen angebaut werden.

\section{F. - ANFLUG VON REBANLAGEN ÜBER GROBE ENTFERNUNGEN}

In den Schadensgebieten im badischen Weinbaugebiet wurde wiederholt festgestellt, daß Bienenstände beeinträchtigt wurden, die weit entfernt von Rebanlagen zur Nutzung der Honigtautracht in den Wäldern standen. In einem Fall stehen mir alle nötigen Unterlagen lückenlos zur Verfügung. Er sei hier als Beispiel angeführt. 
Zwei Wanderstände des Imkers S im Schwarzwald nördlich von Freiburg erlitten offensichtlich Giftschäden. Als Ursache wurden Pflanzenschutzmaßnahmen im Weinbau vermutet, doch waren die nächstgelegenen Rebanlagen $3.5 \mathrm{~km}$ (Luftlinie) entfernt. Im Haarkleid eingesandter toter Bienen und im Darminhalt sowie in einer eingesandten Pollenwabe konnte Vitis-Pollen in großen Mengen nachgewiesen werden. Die beiden Stände waren in einem großen geschlossenen Waldgebiet, etwa 350 und $400 \mathrm{~m}$ über NN, aufgestellt. Nur in westlicher Richtung (Distanz $3.5 \mathrm{~km}$ ) liegen Rebanlagen. Sie grenzen unmittelbar an den Wald. Zwischen den Weinbergen (250-270 m über NN) und dem Bienenstand erstreckt sich ein Höhenrücken, der 519 m Höhe erreicht und den die Bienen überfliegen müssen, wenn sie die Rebanlagen aufsuchen wollen.

Nach Percival (1947) sammeln die Bienen Pollen nur im näheren Umkreis (400 m Radius) ihres Stockes und lassen sich auch durch ein reiches Pollenangebot außerhalb dieses Bereichs nicht von ihrem Verhalten abbringen. Es besteht kein Anlaß, die Richtigkeit dieser Befunde, die in Cardiff/Wales gewonnen wurden, zu bezweifeln. Sie können aber offenbar nicht als allgemeine Regel dienen. Spezielle Situationen bringen die Bienen offensichtlich dazu, ihre Sammelgewohnheiten zu ändern.

Eingegangen im Mai 1977.

Reçu pour publication en mai 1977.

\section{DANK}

Das Ministerium für Ernährung, Landwirtschaft und Umwelt des Landes Baden-Württemberg hat in dankenswerter Weise diese Arbeit zusammen mit anderen Projekten aus dem gleichen Themenkreis gefördert. Herr Dr. Sauter im gleichen Hause stellte freundlicherweise das Zahlenmaterial über den Umfang der Rebflächen und über den Stand der Flurbereinigung zur Verfügung.

Mein Dank gilt auch den zahlreichen Kollegen und Imkern, die das Untersuchungsmaterial beschafft und eingesandt haben, besonders Herrn Dr. R. BuchNen, Freiburg, und seinem Mitarbeiter, Herrn F. Perschil, sowie den Bienenzuchttechnikern Herrn H. Göhringer, Herrn K. Prefferle und Herrn O. Frlenbusch ( + ).

Meinen Mitarbeitern Frau B. Gysler, Frau D. Friedl, Frau U. Krukenbeng, Frau M. Scrwenn und Frau M. Weiß habe ich für die Mithilfe bei der großen Zahl von Einzeluntersuchungen zu danken und Frau H. Magenau für die Durchsicht der Untersuchungsprotokolle auf Vitis-Befunde und die Zeichnung der Abbildung.

Herr Prof. G. Allweld vom Institut für Obst-, Gemüse- und Weinbau gestattete entgegenkommenderweise die Benützung des Versuchsweinbergs. Sein Mitarbeiter, Herr Dr. H. Gebring, hat mit Rat und Tat zu dieser Arbeit beigetragen, desgleichen Herr Dr. G. Schruft vom Weinbauinstitut Freiburg.

\section{RÉSUMÉ}

Jusqu’à ces dernières années on estimait en République fédérale allemande, que les fleurs de vigne (Vitis spp.) n'étaient pas visitées par les abeilles. Des pertes sévères dues aux mesures phytosanitaires prises dans les vignobles obligèrent à reconsidérer le problème. 
On a pu prouver par divers moyens la visite des fleurs de vigne par les abeilles :

1 - par le pollen de Vitis présent dans les poils et dans le tube digestif des abeilles tuées par les produits phytosanitaires répandus dans les vignobles;

2 - par le pollen de Vitis trouvé dans les réserves de pollen;

3 - par les expériences utilisant des trappes à pollen dans les régions de vignoble des cantons du Bad septentrional, du Pfalz et de la vallée du Rhin;

4 - par le pollen de Vitis trouvé dans le miel;

5 - par des observations en champ.

On n'a pas pu établir durant les 6 années d'observations dans le vignoble de Hohenheim s'il y avait ou non sécrétion de nectar.

La récolte de pollen est généralement si peu intensive qu'elle ne se remarque qu'au prix d'une longue observation. Elle n'atteint une fréquence ćlevée, 28 abeilles maximum par cep de vigne et par heure, que quelques jours. Le butinage a lieu principalement dans la matinée.

Les expériences avec des trappes à pollen montrent que les vignes fournissent à certains endroits et pendant un court temps la majeure partie du pollen récolté.

En 1970 la visite des vignes a été particulièrement forte; c'est ce qui résulte de l'exploitation des études de miels au microscope.

En plus des abeilles domestiques, les vignes sont également visitées par des espèces de Bombus, des abeilles solitaires et des syrphes (Syrphidés).

La production de pollen par inflorescence (grappe de fleurs) de la variété Bacchus se situe aux alentours de $1 \mathrm{mg}$; la producgion d'un hectare s'élève donc à $16 \mathrm{~g}$.

Malgré le faible rendement on peut prouver que des vignes sont visitées par des abeilles qui viennent de plusieurs kilomètres. Ceci est en contradiction avec les données bibliographiques, selon lesquelles les abeilles ne récoltent du pollen que dans un faible rayon à partir de leur ruche.

Vitis vinifera est, sous les conditions climatiques et géographiques de la République fédérale allemande, une plante pollinifère de très faible valeur, parfois, néanmoins, d'importance secondaire.

\section{LITERATUR}

Battaglini Marcella und Ricciardelli d'Albore G. C., 1970. - Sulla fora pollinifera de alcune zone dell'Umbria. Note appunti sperimentali entomologia agraria.

Buchner R. O., 1970. - Ein katastrophales Bienensterben im Regierungsbezirk Südbaden. Allg. deutsch. Imkerztg., (9), 223-224.

Geinitz B., 1935. - Bienenverluste durch Arsenmittel. Deutsch. Imkerführer 9 (2), 54-62.

Louveaux J., 1958. - Recherches sur la récolte du pollen par les abeilles (Apis mellifica L.). Ann. Abeille, 3, 113-221.

Louveaux J., Maurizio Anna, Vorwohl G., 1970. - Methodik der Melissopalynologie. Apidologie 1 (2), 193-209.

Lukoschus F., 1957. - Quantitative Untersuchungen über den Pollentransport im Haarkleid der Honigbiene. Z. Bienenforschung, 4 (1), 3-21.

Maurizıo Anna, 1953. - Weitere Untersuchungen an Pollenhöschen. Beih. Schweiz. Bienenztg. $2(20), 485-556$.

Maurizio Anna, 1956. - Pollenanalytische Beobachtungen (15). Ber. Schweiz. Bot. Ges., 66, $127-130$.

MCGREgor S.E., 1976. - Insect pollination of cultivated crop plants. US Department of Agriculture, Washington. 
Percival M., 1947. - Pollen collection by Apis mellifica. New Phytol. 46 (1), 142-173.

Sharples G. C., Todn F. E., MgGregor S. E., Milne R. L., 1965. - The importance of insects in the pollination and fertilization of the Cardinal Grape. Proc. Americ. Soc. Hortic. Sci., 86, 321-325.

STEShenko F. N., 1958. - The role of honeybees in crosspollinating grape vines. Pchelovodstvo 35 (5), 37-40, in russ. Sprache, s.a. Apic. Abstr. $182 / 60$, s.a. Deutsche Bienenwirtsch., 9 (8), $147-148,1958$.

Vorwohl G., 1970. - Bienen (Apis mellifica L.) und Reben (Vitis spp.) Allg. deutsch. Imkerztg., (9), 224-225.

Vorwohl G., 1973. - Drei Jahre Untersuchungen über die Reben als Bienenweidepflanzen. Allg. Deutsch. Imkerztg., (3), 144-146.

VorwoHL G., 1974. - Die Beziehungen zwischen Biene (Apis mellifica) und Reben (Vitis spp.). 24. Intern. Bienenzüchterkongreß Buenos Aires 1973. Apimondia-Verlag Bukarest.

Vorwoнl G., 1977. - Sind die Reben Bienenweidepflanzen? Die Biene, 113 (5), 173-181.

Anschrift des Verfassers : Dr. G. Vorwohl, Universität Hohenheim, Landesanstalt für Bundesrepublik Deutschland. 